\title{
¿Cómo lo medimos? Siete contextos de indagación para detectar y corregir concepciones erróneas sobre magnitudes y unidades
}

\author{
Jordi Domènech Casal \\ Grupo LIEC, Departamento de Didáctica de la Matemática y las Ciencias Experimentales, Universitat \\ Autònoma de Barcelona. \\ IES Vilanova, Vilanova del Vallès (Barcelona) jdomen44@xtec.cat
}

[Recibido en abril de 2013, aceptado en abril de 2014]

\begin{abstract}
Las actividades convencionales de enseñanza de la física sobre magnitudes y unidades pueden tener dificultades en evidenciar o corregir las concepciones erróneas del alumnado. Para responder a esta necesidad, se ha desarrollado una actividad de indagación en el laboratorio partiendo de varios enigmas propuestos al alumnado. Se describe la aplicación de la actividad y se valoran los resultados, junto con el uso de andamios didácticos de apoyo para la escritura creados a propósito para promover la las habilidades científicas del alumnado.
\end{abstract}

Palabras clave: indagación; magnitudes; medidas indirectas; andamios didácticos, física.

How can we measure it? Seven inquiry contexts to detect and correct misconceptions about units and magnitudes

Physics learning activities can present difficulties to detect and correct students misconceptions about magnitudes and units. To this end, we have set up a didactic sequence based on inquiry-based science education approaches where students are asekd to solve several enigmas and design strategies to perform measurements. Results are discussed together with comments about a didactic scaffold used to promote the scientific skills of the students.

Keywords: inquiry; magnitudes; indirect measurements; didactic scaffolds; physics.

\section{Introducción}

En el aprendizaje de las ciencias (y en especial de la física y la química), las magnitudes y unidades son un concepto transversal con el que el alumnado tropieza a menudo (Barragán y Cerpa, 2009). Los problemas teóricos estandarizados, modelos analógicos y los ejercicios de factores de conversión pueden ser insuficientes para manifestar las concepciones erróneas del alumnado o corregirlas, ya que reproducen contextos cerrados que no implican conflicto conceptual en el alumno o no aseguran la existencia de un significado compartido (Moro et al, 2007). Más que problemas de cálculo, el alumnado tiene dificultades para aplicar al mundo real o extraer de él magnitudes y unidades (Ezquerra, 2012, Barragán y Cerpa 2009). Esto se evidencia cuando alumnos perfectamente capaces de calcular la densidad de un cuerpo o de cambiar de unidad de $\mathrm{Km} / \mathrm{h}$ a $\mathrm{m} / \mathrm{s}$ son incapaces de re-contextualizar y representar con sus manos cuánto espacio ocupa un litro o dar una respuesta correcta a la tradicional adivinanza del kilo de paja y el kilo de plomo.

Los trabajos prácticos de laboratorio son una oportunidad para poner en contacto más directo al alumnado con los conceptos científicos (Ezquerra et al, 2012), si bien varios autores alertan que es necesario evitar trabajos puramente manipulativos/reproductivos en los que los alumnos aplican recetas para aprender técnicas con escaso valor cuando se trata de transferirlas a otros contextos (Hodson, 1994, Pedrinacci et al, 2012). En cambio, se defiende en la literatura la utilidad de actividades y contextos didácticos que promuevan el desarrollo de habilidades de razonamiento y actitudes científicas, como propone el modelo de Enseñanza de 
las Ciencias Basada en la Indagación (ECBI) (Caamaño, 2004 y 2012, Barolli et al, 2010, Llewellyn, 2005), entendiendo como contextos el conjunto formado por la actividad didáctica, los materiales, y el entorno (dinámica de aula, concepciones previas...).

En contraste sobre las estrategias de enseñanza de la Ciencia como indagación (Naturaleza de la ciencia), las estrategias de enseñanza mediante la indagación (o ECBI) persiguen un rol más activo del alumnado, que debe aplicar estrategias científicas (emisión de hipótesis, diseño de experimentos, análisis de datos,...) para aprender ciencias (Hodson, 1994, Fernández et al, 2002, Caamaño, 2012), y se estructuran en varias etapas: 1) Formular preguntas investigables 2) Dar prioridad a las evidencias 3) Analizar las evidencias 4) Formular una explicación basada en las evidencias 5) Conectar la explicación con el conocimiento científico 6) Comunicar y justificar la explicación 7) Reflexionar sobre el proceso y el aprendizaje.

Dentro de este marco, el uso de andamios didácticos lingüísticos para promover el desarrollo de habilidades de razonamiento cognitivo-lingüísticas (describir, justificar, argumentar) ha sido propuesto por varios autores como una estrategia útil (Sanmartí, 2003) y aplicado anteriormente en nuestro centro educativo (Domènech, 2013a) para estructurar el razonamiento científico del alumnado.

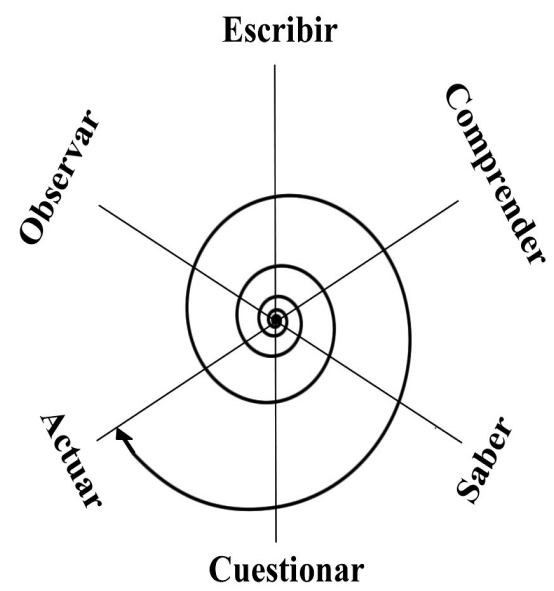

Figura 1. Saber, comprender y escribir son actividades que se, aplicadas secuencialmente, permiten reformular los hechos con la gramática personal que configuran nuestras experiencias y conceptos previos. Modificado de Domènech, 2013b.

La actividad que presentamos en este artículo tiene dos objetivos:

- detectar cualitativamente y mediante actividades de indagación qué tipo de concepciones erróneas manifiesta el alumnado sobre las magnitudes y unidades básicas de volumen, capacidad, longitud y superfície y contribuir a corregirlas.

- mejorar las habilidades de razonamiento científico mediante el uso de andamios lingüísticos.

La actividad se presenta junto con comentarios sobre las situaciones de interés didáctico producidas durante su aplicación.

\section{Descripción de la experiencia}

La experiencia se ha aplicado durante tres cursos académicos (2009-2012) con un total de 180 alumnos de $2^{\circ}$ de ESO del Institut Marta Mata, de Montornès del Vallès. Dura 4 sesiones de clase en el laboratorio, y se ha realizado en paralelo con el trabajo sobre factores de conversión 
en el aula convencional. El alumnado ha participado previamente en actividades ECBI y había trabajado el curso anterior el concepto de densidad y estrategias para su medida en el laboratorio, y las diferentes fórmulas para calcular el área, el volumen y el perímetro de las formas geométricas básicas.

\section{Una actividad sencilla para razonamientos complejos}

Se propone al alumnado que -en equipos de 3 alumnos- resuelva siete enigmas, todos ellos implicando la medida indirecta de alguna magnitud, con varias soluciones posibles, y varias restricciones:

v Los resultados deben ser la media de tres medidas (réplicas) exactas (no aproximadas).

$\checkmark$ Pueden usar sólo los objetos proporcionados (Figura 2).

v El enigma debe resolverse manipulando, pensando y discutiendo entre miembros del equipo. Pueden consultar Internet o libros de texto.

Los 7 enigmas propuestos no tienen un orden de realización, e incluyen determinar:

- La longitud de un ovillo de alambre (sin desenrollarlo)

- La densidad de una roca

- La superficie del área de corte de un alambre

- La superficie de una forma irregular de papel

- El volumen de un paquete de 500 hojas

- El número de clavos de un puñado de clavos (sin contarlos)

- El grosor de un papel

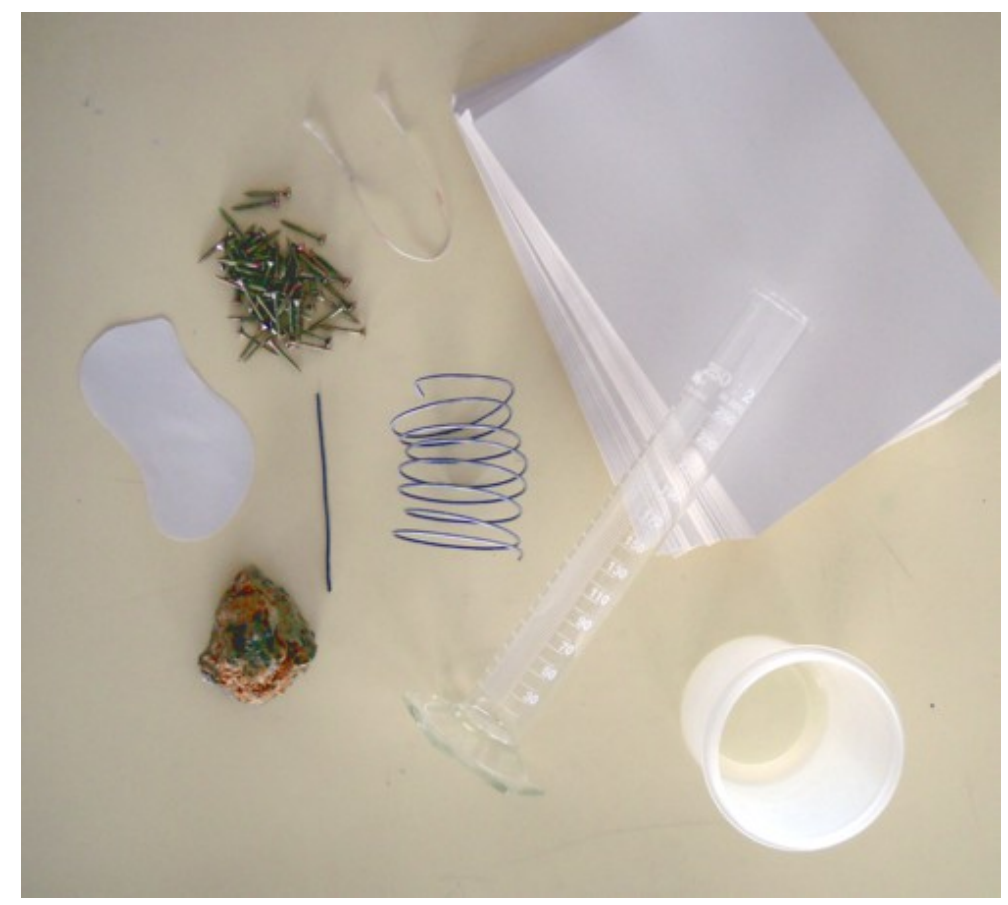

Figura 2.-Los materiales necesarios para la actividad son sencillos, y de manipulación fácil y segura: Un alambre enrollado, otro del mismo tipo recto, una probeta graduada, un recipiente grande no graduado, hojas de papel, un fragmento de papel recortado con forma irregular, un puñado de clavos, un cordel y una piedra (que debe caber en el recipiente grande, pero no en la probeta). A su disposición, regla, agua, y balanzas (analítica y granataria). 
Todas las mediciones necesitan la elaboración de alguna norma lógica de inferencia o extrapolación dentro de una magnitud o entre magnitudes y necesitan del uso estratégico de los conceptos de medición.

Durante las tres primeras sesiones, el alumnado trabaja libremente y el profesor circula por el laboratorio, observando y registrando los incidentes. Ante las consultas del alumnado el profesor se ciñe a ayudas limitadas (ofrecer una pista -predeterminada- o bien reformular la pregunta que hace el alumnado), sin ofrecer las soluciones, ni confirmar si las halladas son correctas.

Confirmar si una respuesta es correcta o no mirando un libro o solucionario elimina la incertidumbre inherente a la investigación (y la vida) real. Educar científicamente debe consistir en empoderar al alumnado para gestionar la incertidumbre. Si en la ciencia real, la máxima certeza la proporcionan las buenas preguntas, los procesos correctos y la construcción social del conocimiento, en la escuela, el profesorado debe centrarse en ayudar a formular buenas preguntas, cuestionar la validez de los procesos y promover las dinámicas de discusión y argumentación. Este enfoque se ha usado anteriormente con el alumnado en otra dinámica llevada a cabo en el centro, las Mistery Boxes (Domènech, 2013b).

\section{Un andamio didáctico para promover la reflexión y la manifestación de concepciones erróneas}

Para cada enigma, los alumnos deben completar una ficha de análisis que contiene varios apartados, atendiendo a las inteligencias múltiples y movilizando distintos lenguajes y habilidades.

\footnotetext{
1. Texto Descriptivo-Argumentativo. Describir la estrategia usada para resolver el enigma. Obligatoriamente, usar al menos 3 de las expresiones siguientes (puedes usar otras que añadir en la lista):

sirve para medir | es directamente proporcional a | se corresponde con | equivale a | se mide en

a continuación | en primer lugar | se calcula mediante

2: Cómic. (Dibujar en formato cómic el proceso seguido, con el máximo detalle. Tener presente que debe poder entenderse sólo mirando el cómic. Para saber si funciona, puedes probar a enseñarlo a alguien que no conozca el proceso y pedirle que te explique qué has hecho).
}

3: Sistematización de resultados: Tabla de Medidas. Para cada medida que hagamos (en algunos enigmas, será necesario hacer varias tablas), hacer una tabla como la siguiente. Siempre que sea posible, mínimo de dos cifras decimales significativas).

\begin{tabular}{|c|c|c|c|}
\hline \multirow{2}{*}{ Magnitud } & Unidad & Medidas & Media \\
\hline \multirow{2}{*}{ Masa } & \multirow{3}{*}{ gramos } & $2,6 \mathrm{gr}$ & \multirow{2}{*}{$2,56 \mathrm{gr}$} \\
\cline { 3 - 3 } & & $2,4 \mathrm{gr}$ & \\
\cline { 3 - 3 } & & $2,7 \mathrm{gr}$ & \\
\hline
\end{tabular}

\section{4: Cálculos.}

5: Texto argumentativo: Conclusiones. Argumenta tus conclusiones, usando como mínimo 2 de los términos siguientes:

por lo tanto | asípues | en consecuencia | puesto que | en cambio | en comparación | proporcionalmente a

Figura 3. La ficha de análisis se estructura en etapas equivalentes a las de los artículos científicos para promover la estructuración del razonamiento científico (Domènech, 2013a).

\section{Pesando superficies: determinar la superficie de un papel de forma irregular}

Se pide al alumno que determine la superficie de una forma irregular de papel (Figura 4). Como solución eficaz, los alumnos calculan la superficie de un folio de forma regular y pesan 
ambos papeles (forma regular y forma irregular) usando la relación de peso como razón de proporcionalidad para calcular la superficie del papel con forma irregular. (asumiendo la misma densidad y grosor para los dos papeles). En la aplicación, algunas situaciones que han evidenciado concepciones erróneas han sido: 1) algunos alumnos intentan delinear con el cordel la forma del papel, argumentando que lo hacen para medir luego la longitud del cordel (confundiendo el perímetro con la superficie) 2) también delineando con el cordel, intentan usar el cordel para, con él, construir una figura con el mismo perímetro y forma cuadrada, para calcular el área (asumiendo una relación constante entre superficie y perímetro).

Cuando ha sido necesario se ha propuesto la pista siguiente: "Si pudiéramos saber la superficie de otro papel de tamaño y forma distinta, ya sólo faltaría saber cuánto más pequeño es el papel del que queremos medir la superficie".

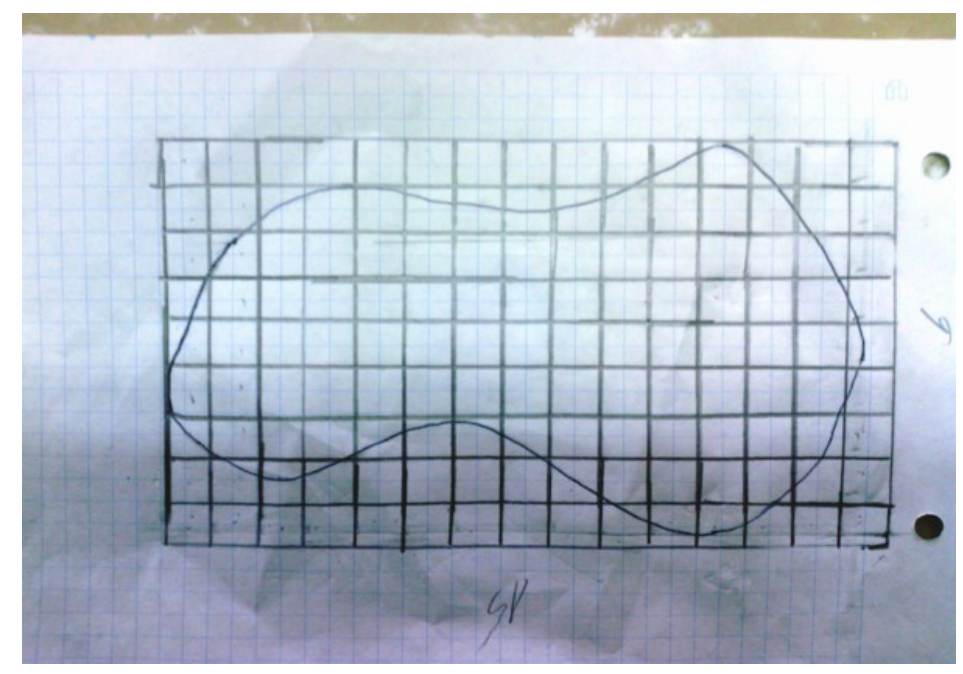

Figura 4.- Intento de aproximar la forma a una composición de áreas calculables, copiando la forma sobre una cuadrícula. En algún caso, los alumnos han añadido rectángulos cada vez más pequeños a la línea curva, en un razonamiento que precede al cálculo integral.

\section{Restando volúmenes: determinar la densidad de una roca}

La modalidad clásica de este ejercicio es conocida por el alumnado, que sabe de experiencias previas que debe medir la masa con la balanza y el volumen por inmersión.

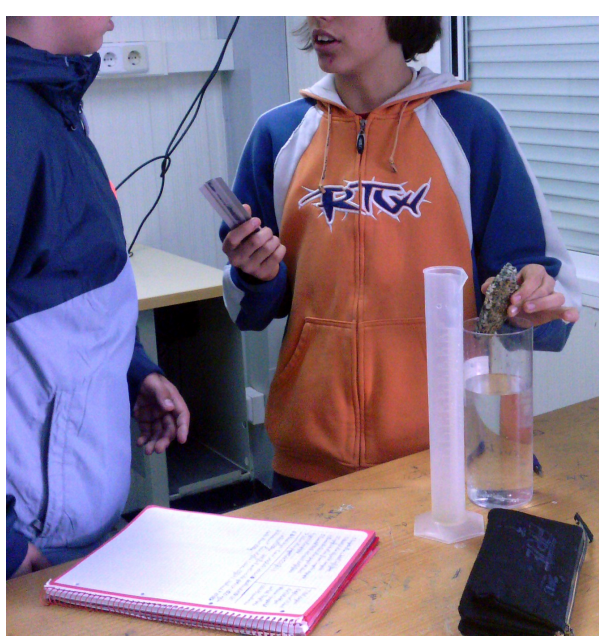

Figura 5. En la imagen, alumnos pactando su estrategia para medir la densidad de un cuerpo de forma irregular.
Construir estrategias alrededor de y mediante las magnitudes y unidades provoca situaciones de negociación de significados en las que los alumnos deben consensuar, no sólo cómo resolver el enigma, sino también los conceptos físicos que participan en él..

En este caso, el alumnado halla una dificultad inesperada: la roca no cabe dentro del recipiente graduado, lo cual implica desarrollar estrategias alternativas con el recipiente no graduado. Como propuesta eficaz, los alumnos marcan en el recipiente no graduado el nivel de agua antes y después de sumergir el objeto, y miden (por adición, mediante la probeta graduada) el volumen de agua que supone este incremento. Una vez conocido el volumen, miden la masa con la balanza y calculan la densidad. 
Se manifiestan concepciones erróneas en dos situaciones: 1) los alumnos miden con la regla el cambio de altura de líquido, y toman la medida en centímetros como si fuera el volumen. 2) los alumnos consiguen realizar medidas indirectas, pero confunden las marcas de $\mathrm{ml}$ de la probeta graduada por $m m$ e intentan aplicar esta "altura" para calcular el volumen mediante la fórmula del cilindro.

\section{3 y 4. Infiriendo proporciones: número de clavos de un puñado de clavos y longitud de un alambre enrollado}

Se especifica al alumnado que no está permitido contar directamente los clavos o enderezar el alambre, que está enrollado en varias vueltas no circulares.

La pista usada para orientar al alumnado ha sido: "no podemos contarlos todos, pero podemos contar algunos y determinar qué proporción suponen"; "podemos medir otro alambre y saber cuánto más grande es el que debemos medir".

El alumnado, en general, ha deducido por si mismo que "cuánto más grande" podía medirse mediante la masa, en muchos casos aplicando lo aprendido en el primer enigma, y usando dos o tres clavos o el fragmento de alambre recto como referencia.

El alumnado ha detectado pequeñas variaciones en el peso de los diferentes clavos, ante lo que a menudo deciden espontáneamente ampliar el número de réplicas más allá de tres. En la mayoría de los casos, el resultado final contenía cifras decimales (cerca de 55,8 clavos), forzando al alumnado a separar los datos (cálculos-resultados) de sus interpretaciones (conclusiones).

\section{5 y 6. Múltiples y divisores. Medir el grosor de un papel y el volumen de un paquete de folios}

Los alumnos perciben rápidamente que es imposible medir el grosor de una hoja directamente con las herramientas proporcionadas, y que la clave está en partir de un número determinado de folios para establecer proporciones después. La pista -única- ofrecida ha sido necesarias en muy pocos casos (¿De cuántos folios podemos determinar el grosor?).

Prácticamente todos los alumnos son capaces de establecer, antes de empezar a medir, una estrategia general que permite usar el resultado de un enigma (el grosor de un folio) para el otro.

Se manifiestan dificultades en identificar el paquete de folios como un prisma, hasta que se dispone un paquete de folios en vertical, seguramente porque esta figura se representa habitualmente de pie.

\section{7-Geometría para medidas imposibles: la superficie de la sección de un alambre.}

En este enigma, con escasos referentes compartidos con los otros, más de la mitad de los alumnos ha necesitado el apoyo de la pista (una vuelta al alambre da una idea de cuán grueso es el alambre, que en el fondo es un cilindro alargado).

Aunque los alumnos detectan de inmediato la forma circular de la sección, y que determinar el radio del alambre es la clave, las dificultades para continuar a partir de ahí, revelan confusiones entre los conceptos de superficie y longitud de la circunferencia. Les es difícil descubrir que pueden usar el cordel como instrumento para medir el perímetro de la sección. Tienen dificultad para recordar las fórmulas matemática del círculo o para trabajar con ellas. El enigma promueve la reflexión, en el sentido que en ocasiones, las medidas indirectas son más 
precisas que las directas; de hecho, deducen que dando varias vueltas alrededor del alambre y dividiendo por el número de vueltas, la precisión mejora cuantas más vueltas se den.

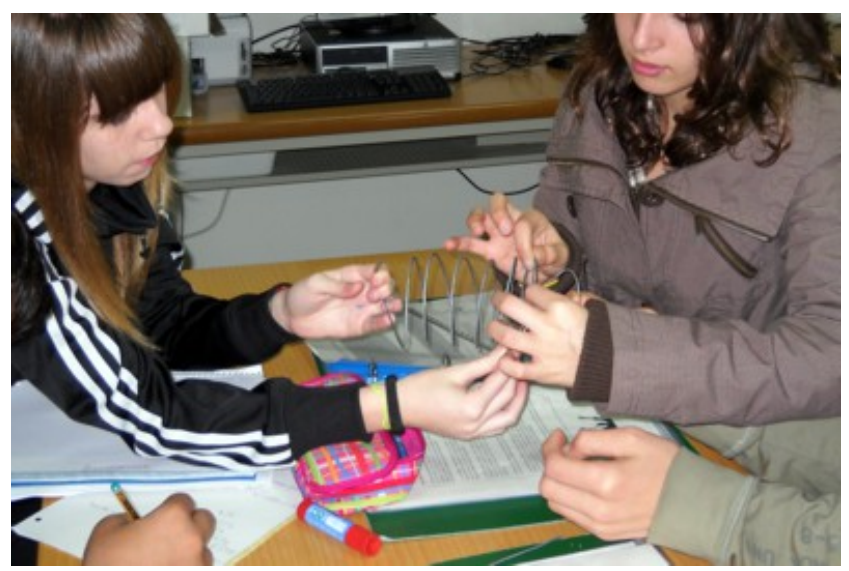

Figura 6. Algunos alumnos intentan medir la longitud del alambre estableciendo el cordel como unidad de medida para después transformar "cordeles" en cm. La inexactitud de los resultados les desanima de usar esta estrategia.

\section{Resultados}

\section{Generar conflictos conceptuales mediante la indagación: una herramienta para identificar y corregir concepciones erróneas}

El estilo problematizador de la actividad ha promovido la instrumentalización de conceptos alrededor de varios contextos (cada uno de los enigmas). Este entorno más complejo que el de los ejercicios cerrados y pre-definidos ha contribuido a evidenciar varias concepciones erróneas (Tabla 1), que se han detectado tanto en la observación en el aula como en la revisión de las fichas de análisis presentadas por el alumnado.

\begin{tabular}{|c|c|c|}
\hline \multirow{4}{*}{$\begin{array}{l}\text { Confusión de } \\
\text { magnitudes }\end{array}$} & Confusiones entre densidad y masa o entre densidad y volumen & Muy frecuente \\
\hline & Medir el volumen en centímetros (por la altura en la probeta) & Frecuente \\
\hline & Confusión entre perímetro y superficie & Muy frecuente \\
\hline & Confusión entre grosor y volumen & Poco frecuente \\
\hline \multirow{3}{*}{$\begin{array}{l}\text { Identificación } \\
\text { de unidades }\end{array}$} & Leer o escribir mm para referirse a $\mathrm{ml}$ & Poco frecuente \\
\hline & $\begin{array}{l}\text { Usar unidades no estándar de medida (medir la altura de un } \\
\text { paquete de folios en folios y no en mm) }\end{array}$ & Poco frecuente \\
\hline & Usar unidades inexistentes ( $\mathrm{ml}$ cúbicos) & Poco frecuente \\
\hline \multirow{4}{*}{$\begin{array}{l}\text { Identificación o } \\
\text { aplicación de } \\
\text { elementos } \\
\text { geométricos }\end{array}$} & Confusión entre radio y perímetro & Poco frecuente \\
\hline & Confusión entre perímetro y área y sus fórmulas & Muy frecuente \\
\hline & $\begin{array}{l}\text { Establecimiento de proporcionalidades directas entre la área y } \\
\text { perímetro de formas geométricas distintas }\end{array}$ & Frecuente \\
\hline & $\begin{array}{l}\text { Dificultades en identificar la forma geométrica del prisma } \\
\text { cuando se encuentra tumbado. }\end{array}$ & Muy frecuente \\
\hline
\end{tabular}

Tabla 1. Descripción semi-cuantitativa de las concepciones erróneas más frecuentes detectadas en el conjunto de los enigmas y corregidas mediante esta actividad. Se ha medido de forma aproximada la frecuencia de cada tipo de error a partir de la observación en el aula y el análisis de las fichas de análisis presentadas por el alumnado. Se consideran "Muy frecuentes" las concepciones erróneas presentadas por más del 30\% del alumnado, "Frecuentes" las presentadas por entre un 10 y un 30\%, y "Poco frecuentes" las presentadas por menos del 10\% del alumnado. 

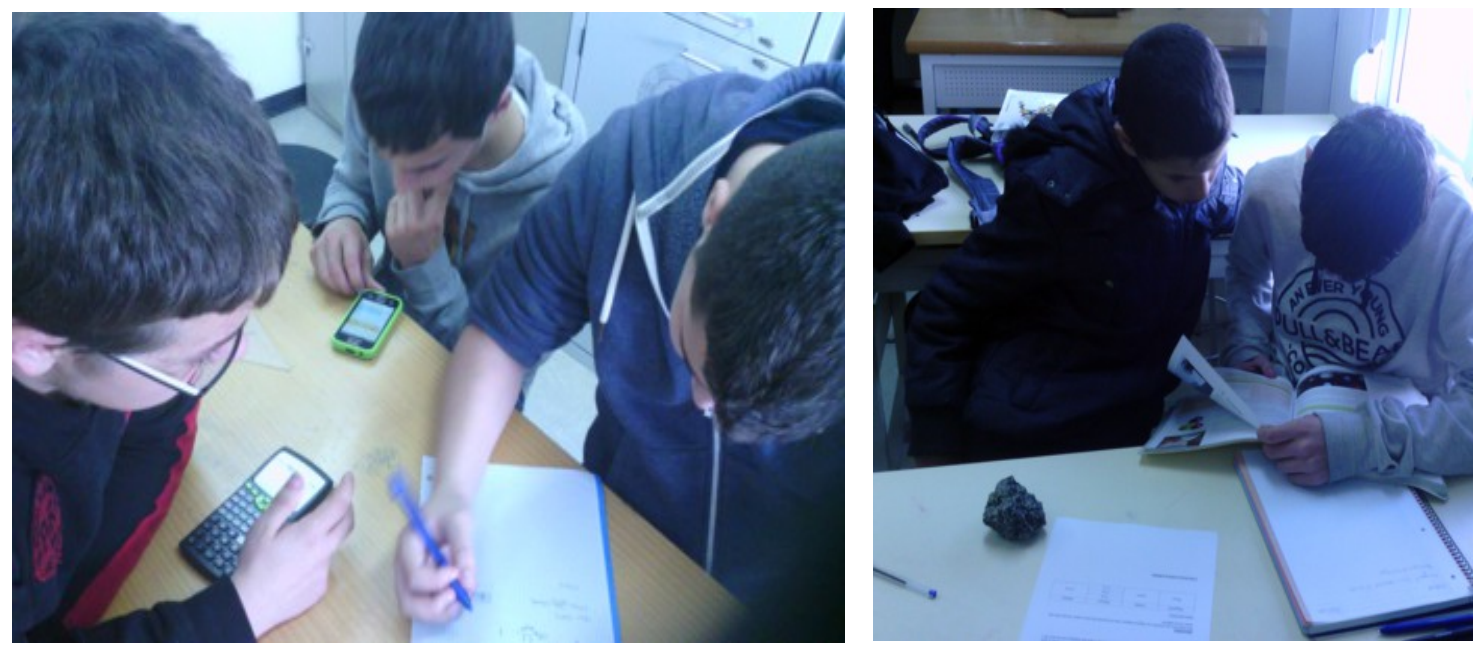

Figura 6. El formato indagador de la actividad promueve la instrumentalización y conjunción de contenidos, habilidades y recursos. a) dos alumnos calculan mientras otro comprueba la fórmula de la densidad mediante el mòvil. b) buscando de motu proprio información en el libro de texto. Esto ha promovido que alumnos que en aula convencional con enfoques tradicionales mostraban baja participación hayan contribuido de manera especial, en algunos casos, liderando sus equipos de trabajo.

\section{Dialogar y escribir para argumentar: construyendo conocimiento y promoviendo el meta-aprendizaje. Resultados del uso de andamios lingüísticos}

Las observaciones en el aula han permitido detectar numerosas situaciones de negociación de significados entre compañeros, para consensuar el significado de términos como "volumen", "densidad", "proporcional", en varias ocasiones haciendo referencia a contenidos de cursos anteriores o incluso programas televisivos. Esto ha llevado a la construcción de definiciones incompletas, pero funcionales en varios contextos (el volumen como "lo que oсирa", la densidad como "lo pesado que es, pero no lo que pesa", la proporción como "lo más grande que es"). Este diálogo no sólo abarca a los alumnos, sino que también incluye diálogos alumnosmateriales: en algún caso, a pesar de no tener una idea clara de la estrategia a usar, algunos equipos han llevado a cabo experimentos "prospectivos" en los que han experimentado manipulando los materiales sin estrategia definida, y de esta interacción ha surgido una primera idea de estrategia.

Completar la ficha de cada enigma ha sido un paso especialmente relevante en la actividad, pues varios grupos han detectado concepciones erróneas o errores de estrategia en este punto.

La redacción de las fichas tiene también por objetivo la promoción de habilidades científicas básicas, que se corresponden con los distintos apartados describir (1), representar o modelar (2), registrar y sistematizar (3), calcular (4) y argumentar (5), aunque es difícil medir un progreso en este sentido en una actividad tan concreta.

Aún así, en la producción escrita, con respecto a experiencias anteriores en el laboratorio, el uso de conectores gramaticales ha aumentado, observándose un mejora en los razonamientos. En el diálogo final de la actividad, una gran parte del alumnado (cerca del 80\%) defiende que argumentar y describir su proceso le ha permitido entenderlo mejor. Mejorar los procesos de indagación orientándolos hacia la comunicación es una vía que otros autores han propuesto con anterioridad (Caamaño, 2002, Menoyo, 2010), y encuentra su base en la conexión íntima entre habilidades cognitivas y lingüísticas, que persigue esta actividad. 
a)

\section{- Primer apartat. Eᄐ text:}

Agafem el pot ple de claus, $\mathrm{i}$ ho pesem a la bàscula per tant $\mathrm{d}$ aconseguir el pes exacte del pot amb els claus, després, agafem tots els claus i ho deixem a part buidant tot el pot, per pesar-ho. Al acabar de pesar el pot buit, agafem un clau i ho pesem sòl en la bàscula. Per això fem servir una bàscula en canvi per el pot fem servir una balança, perquè ges pesuival a molta diferència per fel el càlcul.

b)

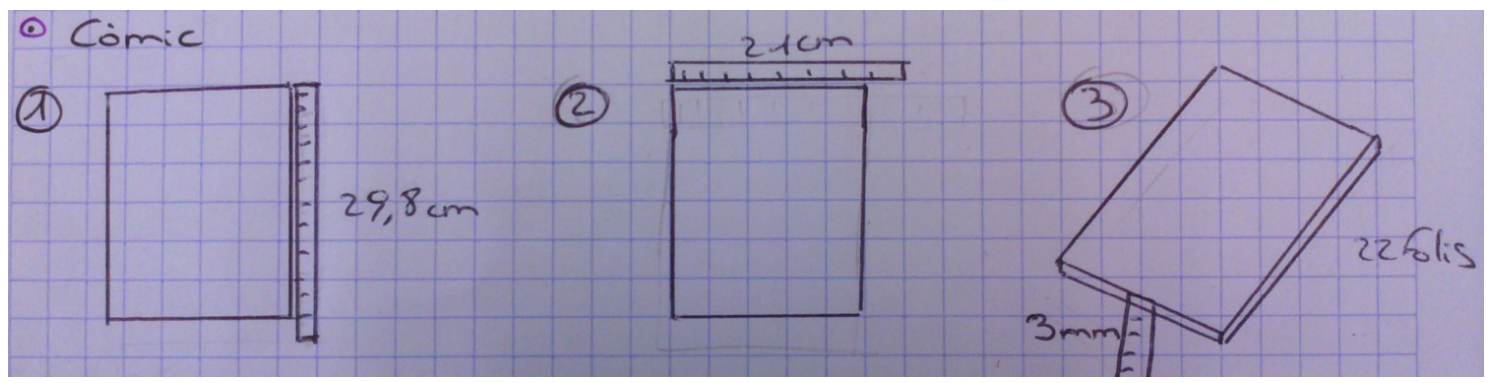

c)

\begin{tabular}{|c|c|c|c|c|}
\hline & MAGNITUD & UNITAT & MESURES & MITJANA \\
\hline $\begin{array}{c}\text { Filferro } \\
\text { petit }\end{array}$ & Massa & Gram & $3.2 / 3.2 / 3.2 \mathrm{~g}$ & $3.2 \mathrm{~g}$ \\
\hline $\begin{array}{c}\text { Llargada } \\
\text { filferro } \\
\text { petit }\end{array}$ & Longitud & Centimetre & $\begin{array}{c}12.2 / 12.2 / 12.2 \\
\mathrm{Cm}\end{array}$ & $12 . .2 \mathrm{~cm}$ \\
\hline $\begin{array}{c}\text { Filferro } \\
\text { gran }\end{array}$ & Massa & Gram & $42.1 / 42.1 / 42.1 \mathrm{~g}$ & $42.1 \mathrm{~g}$ \\
\hline
\end{tabular}

Figura 7. Muestras de distintas partes de fichas de distintos enigmas producidas por los alumnos (en catalán). a) Texto descriptivo-argumentativo, en el que el alumnado usa (marcados en el texto) algunos de los conectores propuestos, como herramientas para un proto-texto instructivo y argumentativo. b) cómic sobre la medida del volumen, en el que se evidencia mediante el dibujo en perspectiva y las distintas posiciones de la regla el concepto de volumen como tridimensional. c) Tabla de datos y cálculos del alumnado, en la que se identifican las magnitudes con las unidades.

En la última sesión, como síntesis de la actividad, las fichas presentadas por cada grupo se han ido comentando en la clase en pequeños grupos de 15 alumnos, en diálogo estructurado con la ayuda de las preguntas: ¿Cómo habéis sabido lo que habéis sabido? ¿Cuán seguros estáis de vuestra respuesta? ¿Porqué? ¿De qué cosas no estáis seguros? ¿Cómo podéis resolver esta duda? ¿Qué errores hay? ¿Porqué se han producido estos errores? Este diálogo metacognitivo (última fase del diseño estándar de las actividades de indagación) ha permitido fomentar la argumentación de las estrategias propuestas, un análisis del proceso de aprendizaje por parte 
del alumnado y corregir las concepciones erróneas detectadas. La actividad ha resultado constituir un buen punto de referencia al que apelar posteriormente "os acordáis cuando...?" y su formato de reto la convierte en una buena opción también para actividades de divulgación científica.

\section{Conclusiones}

Este estudio ha permitido detectar algunas concepciones erróneas sobre magnitudes y unidades que pueden estar inconscientemente siendo "sobrevoladas" en las clases no manipulativas o de carácter demostrativo. Consideramos que la combinación de carácter indagador y manipulador de la experiencia (hands-on minds on) ha contribuido a evidenciar esas concepciones erróneas y constituye una vía para mejorar la enseñanza de conceptos abstractos en las ciencias.

El uso de actividades de comunicación y andamios didácticos lingüísticos para mejorar las habilidades de razonamiento del alumnado ha sido descrito anteriormente por otros autores (Sanmartí, 2003, 2008) y otras experiencias previas en el mismo centro educativo (Domènech, 2013a), y constituye un paso clave en los procesos de enseñanza por indagación, además a una aportación valiosa a la comprensión, por parte de los alumnos, de los objetivos y procesos.

Consideramos de especial importancia la transferencia que se observa de ideas y razonamientos de un enigma a otro que realizan los alumnos, dado que el aprendizaje significativo se produce cuando el alumnado es capaz de transferir los razonamientos a nuevos contextos, como otros autores ya han descrito previamente (Pozo y Gómez, 2010). El lector encontrará en este sentido de interés experiencias de otros autores sobre el aprendizaje contextualizado sobre magnitudes y unidades (Matusevich, 2009, Sierra et al, 2008, López, V., 2004).

\section{Agradecimientos}

La reflexión sobre la experiencia se inscribe en el trabajo del grupo de investigación LIEC (Llenguatge i Ensenyament de les Ciències) del Departamento de Didáctica de la Matemática y las Ciencias Experimentales de la Universidad Autónoma de Barcelona, grupo de investigación consolidado (referencia 2009SGR1543) por AGAUR (Agencia de Ayudas Universitarias y de de Investigación) y financiado por la Dirección General de Investigación, Ministerio de Educación y Ciencia (referencia EDU-2012-38022-C02-02). El autor quiere expresar su agradecimiento a la profesora Núria Domènech Puigdemont sus sugerencias y aportaciones al desarrollo de esta experiencia.

\section{Referencias bibliográficas}

BARRAGÁN, A.L., CERPA, G. (2009). Cocientes y unidades: ¿¿Qué comprenden realmente los estudiantes de física de nuevo ingreso a la universidad?. Revista Eureka sobre Enseñanza y Divulgación de las Ciencias, 6(3), 387-395.

BOGNER, F., BOUDALIS, A., SOTIROU, S. (Eds.) (2012). Pathway. Best Practices of InquiryBased Science Education. Methods and Activities. Epinoia, Pallini Attikis, Greece.

BYBEE R. W. (2006). Scientific Inquiry and Science Teaching. En Flick, L. B., Lederman, N. G. (Eds.), Scientific Inquiry and Nature of Science. Implications for Teaching, Learning and Teacher Education. (pp. 1-12). Dordrecht, The Netherlands: Springer.

CAAMAÑO, A. (2002). ¿Cómo transformar los trabajos prácticos tradicionales en trabajos prácticos investigativos? Aula de Innovación Educativa, 113, 21-26 
CAAMAÑO, A. (2004). Experiencias, experimentos ilustrativos, ejercicios prácticos e investigaciones: Una clasificación útil de los trabajos prácticos? Alambique: didáctica de las ciencias experimentales, 39, 8-19.

CAAMAÑO, A. (2012). ¿Cómo introducir la indagación en el aula? Los trabajos prácticos investigativos. Alambique: didáctica de las ciencias experimentales, 70, 83-91.

DOMÈNECH, J. (2013a). Secuencias de apertura experimental y escritura de artículos en el laboratorio: un itinerario de mejora de las prácticas de laboratorio. Enseñanza de las Ciencias, 31(3), 249-262

DOMÈNECH, J. (2013b). Escritura de artículos y diseño de experimentos: andamios para escribir, pensar y actuar en el laboratorio. Enseñanza de las Ciencias, número especial. Congreso ENSE Ciencias 1085-1089.

DOMÈNECH, J. (2013c). Les Mistery boxes, una activitat senzilla d'indagació a l'aula com a metàfora de la ciència. Ciències 24, 20-25

EZQUERRA, A., ITURRIOZ, I., DÍAZ, M.(2012). Análisis experimental de magnitudes físicas a través de vídeos y su aplicación al aula. Revista Eureka sobre Enseñanza y Divulgación de las Ciencias, 9(2), 252-264.

FERNÁNDEZ, I., GIL, D., CARRASCOSA, J., CACHAPUZ, A., PRAIA, J. (2002). Visiones deformadas de la ciencia transmitidas por la enseñanza. Enseñanza de las Ciencias, 20 (3), 477-488.

HODSON, D. (1994). Hacia un enfoque más crítico del trabajo de laboratorio. Enseñanza de las Ciencias, 12(3), 299-313

LÓPEZ, V. (2004). La física de los juguetes. Revista Eureka sobre Enseñanza y Divulgación de las Ciencias, 1(1), 17-3

LLEWELLYN, D. (2005). Teaching High School Science through Inquiry: A case study approach. Corwin Press \& NSTA press.

MATUSEVICH, M.A. (2009). La relación superfície-volumen como ventaja selectiva. Secuencia de actividades didácticas. Revista Eureka sobre Enseñanza y Divulgación de las Ciencias, 6(2), 278-286

MENOYO, M.P. (2010). ¡Yo me apunto a hacer trabajos de investigación! La voz del profesorado y el alumnado. Aula de Innovación Educativa, 195, 56-62

MORO, L.E., VIEU, J.E., ZAMORANO, R.O., GIBBS, H.M. (2007). Aprendizaje de los conceptos de masa, peso y gravedad. Investigación de la efectividad de un modelo analógico. Revista Eureka sobre Enseñanza y Divulgación de las Ciencias, 4(2), 272-286

PEDRINACCI, E., CAAMAÑO, A., CAÑAL, P., DE PRO, A. (2012). 11 ideas clave. El desarrollo de la competencia cientifica. Graó, Barcelona

POZO, J. I., GÓMEZ, M. A. (2010). Por qué los alumnos no comprenden la ciencia que aprenden. Alambique, Didáctica de las Ciencias Experimentales, 66, 73-79.

OSBORNE, J., DILLON, J. (2008). Science Education in Europe: Critical Reflections. Report to the Nuffield Foundation.

ROCARD, M., CSERMELY, P., JORDE, D., LENZEN, D., WALWERG-HERIKSSON, H., HEMMO, V. (2006). Science Education Now: a new pedagogy for the future of Europe. Report for the European Comission. 
SANMARTÍ, N. (2008). Escribir para aprender ciencias. Aula de Innovación Educativa, 175, 2932.

SANMARTÍ, N. (Coord.) (2003). Aprendre ciències tot aprenent a escriure ciència. Edicions 62: Barcelona.

SIERRA, D., GARCÍA, G, DELGADO, M., MORA, C., CHOURIO, A. (2008). Midiendo el diámetro de la Tierra. Revista Eureka sobre Enseñanza y Divulgación de las Ciencias, 5(3), 293-301. 